\title{
Moderate hypofractionated radiotherapy after prostatectomy for cancer patients: toxicity and clinical outcome
}

This article was published in the following Dove Press journal: Cancer Management and Research

\author{
Salvina Barra' \\ Liliana Belgioia ${ }^{1,2}$ \\ Michela Marcenaro' \\ Serena Callegari ${ }^{2}$ \\ Alice Pastorino ${ }^{2}$ \\ Luca Trapani \\ Francesca Cavagnetto 3 \\ Stefania Garelli ${ }^{3}$ \\ Renzo Corvò ${ }^{1,2}$ \\ 'Radiation Oncology Department, \\ Ospedale Policlinico San Martino, \\ Genoa, Italy; ${ }^{2}$ Department of Health \\ Sciences (DISSAL), University of \\ Genoa, Genoa, Italy; ${ }^{3}$ Medical Physics \\ Department, Ospedale Policlinico \\ San Martino, Genoa, Italy
}

Background: After radical prostatectomy (RP) radiotherapy (RT) plays a role, both as adjuvant or salvage treatment. If negative features are present such as extracapsular extension, seminal vesicle invasion, lymph invasion, and positive surgical margins, RT after RP reduces the risk of recurrence, although it is associated with an increased risk of acute and late toxicities. An intensified RT delivered in a shortened time could improve clinical outcome and be safely combined with hormonal therapy (HT). The aim of this study was to determine the acute and late toxicities associated with hypofractionated RT and to assess the impact of the addition of HT to RT in high-risk prostate cancer (PC) patients on overall response and toxicity.

Materials and methods: Sixty-four PC patients undergoing RP were included in this retrospective study. All patients were recommended to receive adjuvant or salvage RT. Prescription doses were 62.5 Gy in 25 fractions to prostate bed, 56.25 Gy in 25 fractions to seminal vesicles bed, and $50 \mathrm{~Gy}$ in 25 fractions to pelvis if indicated. HT was administered to patients with additional adverse pathologic features including Gleason score $>7$, prostate-specific antigen $>20 \mathrm{ng} / \mathrm{mL}$ before surgery, or prostate-specific antigen with rapid doubling time after relapse or nodal involvement. After completion of RT, patients were observed after 4 weeks, and then followed-up every 3-6 months. Acute and late toxicities were assessed using Common Terminology Criteria for Adverse Events v4 and Radiation Therapy Oncology Group scale, respectively. Results: For acute toxicity, only grade 1 gastrointestinal and genitourinary toxicities were detected in $17 \%$ and $11 \%$ of patients, respectively. As regards late toxicity, only $5 \%$ of the patients developed grade 1 gastrointestinal adverse event; grade 1, grade 2 , and grade 3 genitourinary toxicity was recorded in $5 \%, 3.3 \%$, and $3.3 \%$ of patients, respectively. Two and 5 years overall survival were $98 \%$ and $96 \%$, respectively. The curves stratified for treatment show a slight difference between patients receiving RT or $\mathrm{RT}+\mathrm{HT}$, but the differences did not reach statistical significance $(p=0.133)$ Conclusion: In patients with PC undergoing RP, hypofractionated RT may contribute to achieve a high overall survival with an acceptable toxicity profile. Combination of RT and HT is also well tolerated and efficacious.

Keywords: prostate cancer, postoperative radiotherapy, hypofractionation

\section{Introduction}

Prostate cancer (PC) represents one of the most frequent cancers worldwide along with breast, lung, and colon cancer. ${ }^{1}$ Patients with localized prostatic cancer are often treated with surgery, but more than $30 \%$ of tumors eventually recur. After surgery, radiotherapy (RT) plays a role, both as adjuvant or salvage treatment, if adverse features are present or if prostate-specific antigen (PSA) levels increase. Large randomized controlled trials addressing the role of postoperative RT have been completed. ${ }^{2-4}$ In
Correspondence: Liliana Belgioia University of Genoa-Ospedale Policlinico San Martino, Largo Rosanna Benzi, 10, 16132 Genoa, Italy

Tel +39010 5558933

Email liliana.belgioia@unige.it 
case of extracapsular extension, seminal vesicle invasion, lymph invasion, and positive surgical margins, RT after RP reduces the risk of recurrence. However, this is associated with an increase in acute and late toxic effects. Prospective studies comparing adjuvant and salvage RT are ongoing, and dose escalation, different RT techniques, and concomitant use of hormonal therapies (HT) are unsolved open questions.

Conventionally, RT is delivered with a standard prolonged fractionation of 2 Gy per single dose. Recently, hypofractionated RT, ie, shortening the overall treatment time by delivering an effective biological dose with single dose higher than $2 \mathrm{~Gy}$, has been shown to maintain the clinical benefits of conventional RT, although it has been reported to be associated with a higher risk of acute and late effects. Thus, gastrointestinal (GI) and genitourinary (GU) toxicities might be the main drawbacks of hypofractionated RT. The biological basis for a hypofractionated RT is that PC, compared to other cancers, has a lower $\alpha / \beta$ ratio ( $\alpha$ and $\beta$ are the parameters of radiosensitivity adopted in modeling formulae of fractionation). ${ }^{5-8}$ Tumors with a low $\alpha / \beta$ ratio may benefit from fractionated schedules with single doses above 2.4-2.6 Gy. ${ }^{9,10}$ Hypofractionated RT, when delivered with advanced technology (Image-guided Radiotherapy [IGRT] and Intensity-modulated Radiotherapy [IMRT]), has the potential to limit GI and GU toxicities, although initial experiences gave controversial outcomes. ${ }^{11,12}$ On a positive note, hypofractionated RT has lower costs in an RT department and is much more convenient for patients.

In locally advanced PC, a significant proportion of patients progress following RT alone regardless of fractionated schedule. ${ }^{13-15}$ In this setting, patients at high risk (ie, those with high Gleason score or high PSA levels) may benefit from the addition of HT, as suggested by several trials and two meta-analyses showing decreased mortality and recurrence with an acceptable toxicity. ${ }^{13-15}$ Recently, a randomized study showed that the addition of antiandrogenal therapy to salvage radiation therapy resulted in higher rates of long-term overall survival (OS) and low incidences of metastasis cancer. ${ }^{16}$

It is still unclear whether the addition of HT to shortened hypofractionated RT provides a good profile of compliance beyond the excellent biochemical disease control and OS.

We here report the results of a retrospective study on the moderate hypofractionated RT after prostatectomy. Our primary aim was to determine the acute and late toxicities associated with hypofractionated RT; second, we analyzed if the addition of HT in high-risk PC patients was associated with a better overall response and toxicity profile compared to those of patients receiving RT alone.

\section{Materials and methods Patients}

Between 2010 and 2016, 64 patients with PC undergoing radical prostatectomy (RP) were recommended to receive adjuvant or salvage RT for adverse pathologic features including extracapsular extension, invasion of the seminal vesicles, and positive margins. Patients underwent pelvic RT if there was presence of positive pelvic nodes or in those with a risk $(>15 \%)$ of pelvic nodal involvement, according to Roach formula. ${ }^{17}$ In addition, RT was recommended in those patients experiencing a postprostatectomy raise in PSA that was undetectable before surgery. Androgen deprivation therapy was administered in the presence of adverse pathologic features including Gleason score $>7$, PSA $>20$ $\mathrm{ng} / \mathrm{mL}$ before surgery, or PSA with rapid doubling time after relapse or nodal involvement. Before RT, all patients underwent multiparametric prostate magnetic resonance imaging. Patients with clinical recurrence in the prostate bed or with clinical positive lymph nodes after surgery were excluded from the study. This retrospective data analysis was approved by the Steering Ethical Committee of the IRCCS Policlinico San Martino, Genoa, Italy. All patients signed a written informed consent.

The main characteristics of the patients are reported in Table 1 .

\section{Treatment}

Patients were treated with Helical Tomotherapy (Accuray, Sunnydale, CA, USA) or volumetric-modulated arc therapy using VMAT technique delivered by a High-Tech Linear Accelerator (Varian RapidArc, Palo Alto, CA, USA). All patients underwent computed tomographic (CT) simulation with a full bladder and empty rectum, in the supine position placed in an appropriate fixation device (Combifix, Civco Medical Solutions, Coralville, IA, USA). CT data sets were sent for contouring on the Eclipse treatment planning system (VarianMedical System, Palo Alto, CA, USA) and then exported using DICOM RT (digital imaging and communication in medicine) format to Tomotherapy Planning System or directly to the treatment with the Trilogy Linear Accelerator. For all patients, Clinical Target Volume 1 (CTV) consisted of prostate bed, CTV2 the seminal vesicles bed, and CTV3 included, obturator, presacral, external and internal lymph nodes to below the aortic bifurcation. The 
Table I Main characteristics of the patients analyzed

\begin{tabular}{|c|c|c|c|c|}
\hline Patient characteristic & All Patients & RT & $\mathrm{RT}+\mathrm{HT}$ & $P$-value \\
\hline Number of patients (\%) & 64 & $33(5 \mid .5)$ & $31(48.5)$ & \\
\hline Number of deaths (\%) & 2 & $0(0.0)$ & $2(6.5)$ & \\
\hline Age at surgery, mean (SD) & 64.9 & $64.7(6.8)$ & $64.2(8.2)$ & 0.995 \\
\hline Gleason score $>7, \mathrm{~N}(\%)$ & $27(42.2)$ & $9(27.3)$ & $18(58.1)$ & 0.013 \\
\hline PSA levels before surgery, $\mathrm{ng} / \mathrm{mL}$, mean (SD) & 11.9 & $8.9(5.5)$ & $11.8(6.7)$ & 0.047 \\
\hline Comorbidity $(<\mathrm{I}), \mathrm{N}(\%)^{\mathrm{a}}$ & 25 & II (33.3) & $14(45.2)$ & 0.332 \\
\hline Positive lymph nodes $(\geq \mathrm{I}), \mathrm{N}(\%)$ & 16 & $0(0.0)$ & $16(51.6)$ & $<0.0001$ \\
\hline
\end{tabular}

Notes: ${ }^{2} \mathrm{Cardiovascular}$ disease, arterial hypertension, bowel disease, diabetes mellitus.

Abbreviations: $\mathrm{HT}$, hormonal therapy; PSA, prostate-specific antigen; RT, radiotherapy.

CTV1 was contoured using Radiation Therapy Oncology Group consensus guidelines, ${ }^{18}$ modified according to surgical and pathologic findings. The planning target volume 1 (PTV1) was obtained adding a margin of $5 \mathrm{~mm}$ posteriorly and $7 \mathrm{~mm}$ in all other directions to CTV1; PTV2 and PTV3 were obtained adding a $5 \mathrm{~mm}$ isotropic margin to CTV2 and CTV3.

Rectum, bladder, femoral heads, large and small bowel, and penile bulb were outlined as organs at risk. The course of radiotherapy consisted of 25 fractions of 2.5 Gy daily for a total dose of 62.5 Gy to PTV1. The volume of the seminal vesicles received a total dose of 56.25 Gy in 25 fractions, 2.25 Gy daily. If pelvic nodes were irradiated, a total dose of 50 Gy in 25 fractions with a single dose of 2 Gy per fraction was delivered.

The dose was calculated with the normalized total dose formula considering a prostate $\alpha / \beta$ ratio of $1.5 \mathrm{~Gy}$. Dose-volume histogram (DVH) goals for the rectum were $V 40 \leq 43 \%, V 50 \leq 32 \%$, and $V 65 \leq 10 \%$. The bladder DVH goals were $V 40 \leq 47 \%, V 55 \leq 27 \%$, and $V 60 \leq 14 \%$. The femoral head DVH goal was $V 20<50 \%$; a constraint to the bowel placed out of the PTV was accepted with a mean dose of $19.8 \mathrm{~Gy}$, while for penile bulb, the median dose was set to $39 \mathrm{~Gy}$. Megavoltage CT by HT or Cone-Beam CT by Trilogy was performed every day before treatment to correct patient setup according to bone and soft tissue anatomy and to take into account the intrafraction variability.

Patients were treated every day following a rectum and bladder institutional protocol that strongly recommended empty rectum and drinking $500 \mathrm{~mL}$ of water 30 minutes before each RT fraction after having emptied the bladder. If patients had unacceptable bladder or rectal filling, the treatment was deferred until correct filling volumes were reached.

HT was started the first day of RT and consisted of gonadotropin-releasing hormone agonists or antiandrogen therapy, according to the physician's decision.

\section{Follow-up and toxicity evaluation}

After completion of RT, patients were observed after 4 weeks, then every 3-6 months for physical examination, PSA measurement, and assessment of toxicity. Acute toxicities were retrospectively graded based on physicians' notes during treatment using the Common Terminology Criteria for Adverse Events version 4.0. Late toxicities were defined as those occurring 6 months after RT and were scored using the Radiation Therapy Oncology Group and European Organization for Research and Treatment of Cancer Scale.

\section{Statistical analysis}

A descriptive analysis was done in which clinical and demographic variables were compared in patients with PC receiving $\mathrm{RT}$ or $\mathrm{RT}+\mathrm{HT}$, after $\mathrm{RP}$.

For categorical variables, the numbers and percentages are reported, while for continuous variables the mean and SD values are considered. The $p$-values were calculated using the $\chi^{2}$ test for the categorical variables and the Kruskal-Wallis test for the continuous variables.

The survival curves, stratified for the different clinical variables, were obtained using the Kaplan-Meier method. The Kaplan-Meier method followed by a multivariate Cox model was used to analyze the toxicity following RT, distinguishing between acute and late toxicity.

\section{Results \\ Patients characteristics}

Sixty four men with median age 68 years (range, 48-82) were treated with hypofractionated RT after prostatectomy. The median follow-up of the entire patient population was 15.5 months (range, 3-64 months).

Thirty two patients were treated with adjuvant RT and 32 with salvage RT. Pre-RT median PSA was $0.205 \mathrm{ng} / \mathrm{mL}$ (range, $0.003-5.46 \mathrm{ng} / \mathrm{mL}$ ) in the adjuvant setting and 0.23 $\mathrm{ng} / \mathrm{mL}$ (range, $0.009-5.48 \mathrm{ng} / \mathrm{mL}$ ) in the salvage treatment. 
Median time between surgery and the start of RT was 7 months (range, 3-149).

Sixteen patients were pathological node stage $(\mathrm{pN}+)(12$ in adjuvant, 4 in salvage group), 18 pathological tumor stage (pT)3a (11 in adjuvant, 7 in salvage), 25 pT3b (16 in adjuvant, 9 in salvage), and 5 microscopic residual disease (R1).

Two patients died of causes unrelated to PC. At the time of analysis, no patients had a biochemical relapse. The OS of the whole study group was $>98 \%$ at 24 months.

Thirty-eight patients were irradiated in the pelvic area, $15(40 \%)$ in the adjuvant and $23(60 \%)$ in the salvage RT group. HT was administered in 18 and 13 patients undergoing, respectively, adjuvant and salvage RT. Gonadotropinreleasing hormone agonists were prescribed in 26 patients (84\%), while 5 (16\%) received antiandrogens. Median HT time was 12 months (range, 1-62 months), and 19/31 patients were still on HT at the time of last follow-up.

Table 1 reports the clinical pathological characteristic of the two patient populations (RT and RT+HT). Mean age at surgery and comorbidity were similar between the two groups. A higher percentage of patients treated with RT+HT had a Gleason score $>8(58.1 \%$ vs $27.3 \%)$ and more frequently had more positive lymph nodes compared to those receiving RT only (21.6\% vs $0 \%$ ); in addition, higher PSA levels before surgery were observed in RT+HT treated patients.

\section{Toxicity evaluation}

As regards acute toxicity, only grade $1 \mathrm{GI}$ and GU toxicities were detected in $11(17 \%)$ and $7(11 \%)$ patients, respectively. About 61 out of 64 patients (95\%) were evaluable for late toxicity; 3 patients were excluded because of follow-up duration being less than 6 months. Three of 61 patients $(5 \%)$ developed grade 1 GI adverse event; grade 1, grade 2, and grade $3 \mathrm{GU}$ was recorded in $3(5 \%), 2(3.3 \%)$, and $2(3.3 \%)$ patients, respectively.

A higher incidence of acute toxicity was observed for patients receiving pelvic irradiation $(p=0.0019)$; a similar trend, although not reaching a statistically significant difference ( $p=0.07)$, was observed for late toxicity.

The overall late toxicity rate at 5 years was $73.1 \%$ (Figure 1A). When the "toxicity-free survival" was stratified for the treatment received, it seems that patients receiving RT+HT had a slightly lower toxicity-free survival compared to those receiving RT only, although the differences were not statistically significant. In fact, the Log-rank test $P$-value comparing RT vs RT+HT was 0.247 (Figure 1B). A similar trend was found for acute toxicity (Figure 1C and D).

The results of the multivariate analysis (Cox model) are reported in Table 2 for the acute toxicity and in Table 3 for the late toxicity. Patients with 1 or more lymph nodes have an increased risk (97 fold) of acute toxicity relative to patients with no positive lymph nodes. Pre-RT PSA values, as well as age, type of treatment (RT or RT+HT), or presence of comorbidities did not impact on the risk to develop acute toxicity. A trend, although not reaching a statistical significance $(p=0.094)$, was observed when presurgery PSA levels are considered: an increase of 1 unit in the PSA value is associated with a $7 \%$ increased risk of acute toxicity.

When late toxicity was considered, again patients with 1 or more comorbidities have a statistically significant lower risk $(96 \%)$ of experiencing late toxicity relative to patients with no comorbidities ( $p=0.024)$. Presurgery or pre-RT PSA values as well as type of treatment (RT or RT+HT) did not impact the development of late toxicity. A trend, although not reaching a statistical significance, was observed when age was considered: an increase of 1 year of age is associated with an increased risk of late toxicity of $17 \%$.

Considering the OS of the 64 patients under study, at 60 months there was a high probability of survival (>96\%) (Figure 2A). Having registered only 2 deaths (all in the group of patients receiving $\mathrm{RT}+\mathrm{HT}$ ), the curves stratified for treatment reported in Figure 2B show a slight difference between patients receiving $\mathrm{RT}$ or $\mathrm{RT}+\mathrm{HT}$, although the differences did not reach statistical significance $(p=0.133)$.

The survival curves stratified for the variables found statistically different between the two groups (RT vs RT+HT) are reported in Figure 2C and D. As can be seen, the presence of at least one positive lymph node reduces the probability to survive (Log-rank test $p<0.001$, Figure $2 \mathrm{C}$ ). In patients with preexisting PSA levels $<10$, a better survival, compared to those patients with PSA values $\geq 10$, was found (Figure 2D, Log-rank test $p=0.071$ ).

\section{Discussion}

Approximately $25 \%$ of patients with PC are primary treated with RP. One third of these patients are likely to show tumor recurrence after surgery. ${ }^{19,20}$ An increasing risk of recurrence is observed in patients with high Gleason score and positive, margins and these patients benefit from a postsurgery RT. In fact, adjuvant RT has been demonstrated to both increase progression-free survival (PFS) in different randomized trials and, in one of these trials, also to increase OS..$^{2-4}$ In these studies, the median RT dose delivered to patients was 60-64 Gy. A retrospective study on 334 patients undergoing adjuvant RT showed that patients treated with $\geq 70$ Gy had significantly improved biochemical PFS and cancer-specific survival rates compared with those receiving $\leq 70$ Gy at median follow-up 
A

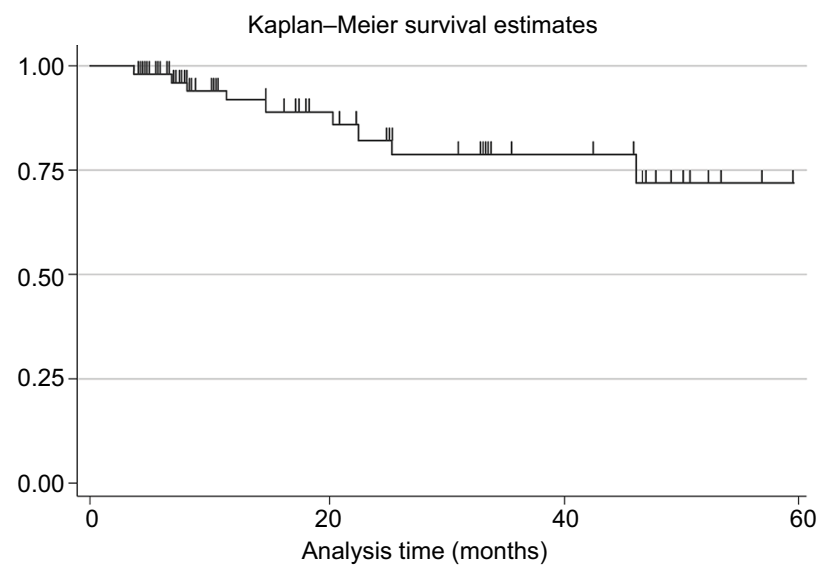

C

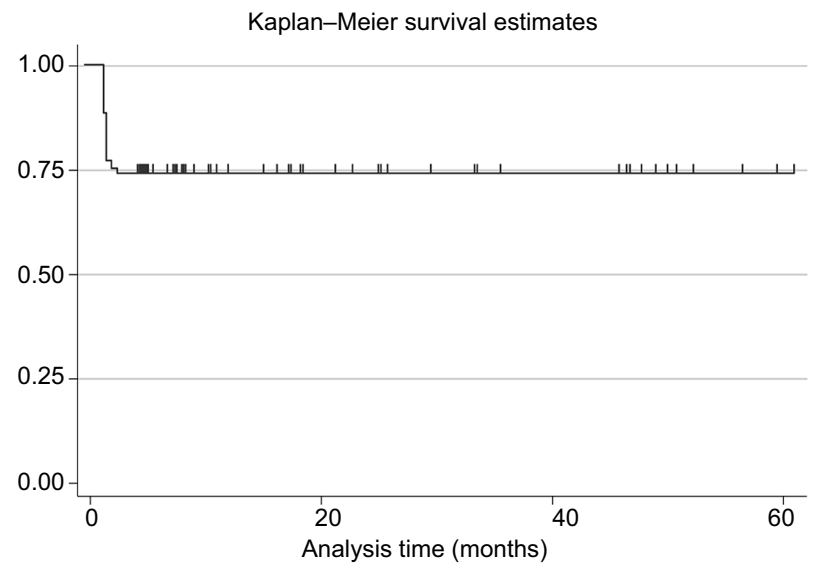

\section{B}

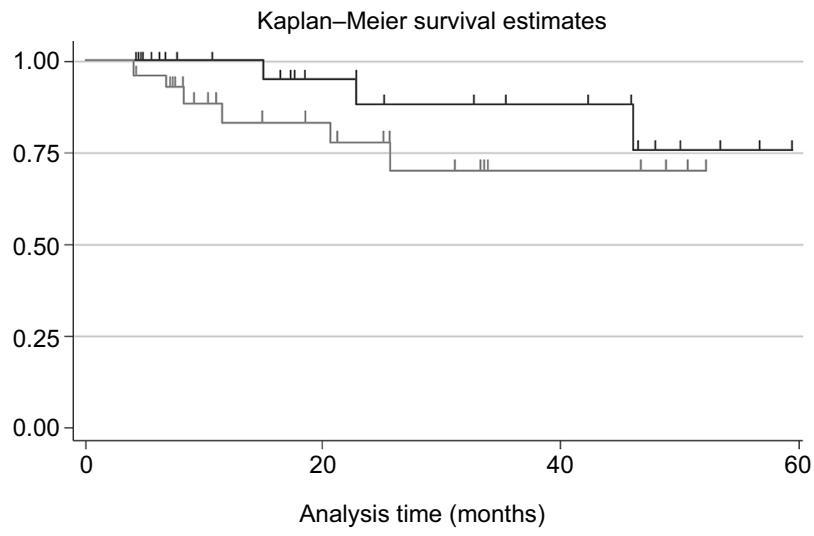

D

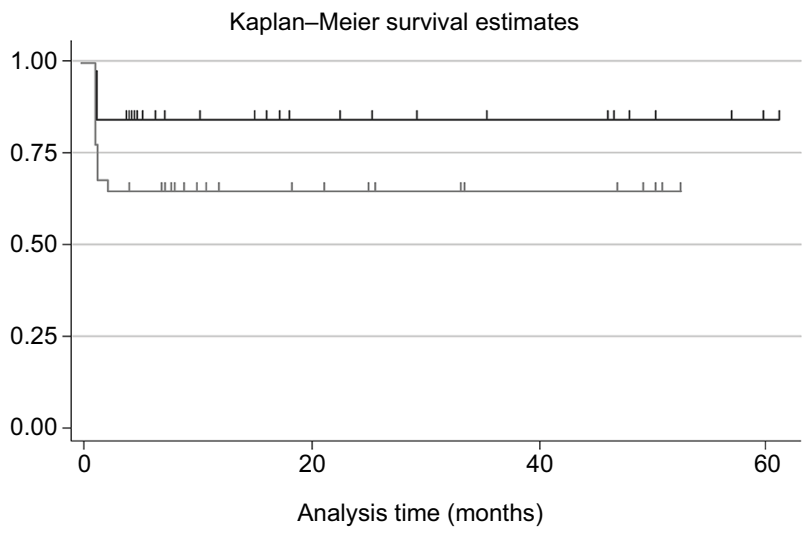

Figure I Kaplan-Meier toxicity-free survival estimates.

Notes: (A) Late toxicity-free survival in the overall population; (B) late toxicity-free survival stratified for treatment received (RT, black line vs RT+HT, gray line); (C) acute toxicity-free survival in the overall population; (D) acute toxicity-free survival stratified for treatment received (RT, black line vs RT+HT, gray line).

Abbreviations: $\mathrm{HT}$, hormonal therapy; RT, radiotherapy.

Table 2 Multivariate analysis (Cox model) for acute toxicity

\begin{tabular}{llllll}
\hline Covariate & HR & SE & $\mathbf{z}$ & $\mathbf{p}>\mathbf{z}$ & 95\% Confidence interval \\
\hline RT+HT & 0.382 & 0.385 & -0.95 & 0.340 & $0.053-2.752$ \\
I+ Comorbidities & 0.913 & 0.742 & -0.11 & 0.910 & $0.185-4.495$ \\
Gleason score $>8$ & 0.206 & 0.180 & -1.81 & 0.070 & $0.037-1.140$ \\
I+ Positive lymph nodes & 19.897 & 24.661 & 2.41 & 0.016 & $1.753-225.81$ \\
RT-salvage & 3.696 & 2.624 & 1.84 & 0.066 & $0.919-14.861$ \\
Pre-RT PSA & 1.433 & 0.281 & 1.83 & 0.067 & $0.975-2.104$ \\
Presurgery PSA & 1.066 & 0.041 & 1.68 & 0.094 & $0.989-1.150$ \\
Age & 1.018 & 0.042 & 0.43 & 0.665 & $0.939-1.103$ \\
Pelvic RT & 9.427 & 10.835 & 1.95 & 0.051 & $0.990-89.689$ \\
\hline
\end{tabular}

Abbreviations: HR, hazard ratio; HT, hormonal therapy; PSA, prostate-specific antigen; RT, radiotherapy; SE, standard error of the mean.

of 108 months..$^{21}$ Other studies confirmed these results, and two systematic reviews reported a $2 \%$ improvement in PFS for each additional Gy delivered..$^{22,23}$ The SAKK 09/10 study is a trial where patients in biochemical failure after prostatectomy but without evidence of macroscopic disease are ran- domly assigned to 64 or $70 \mathrm{~Gy}$, and preliminary results show that dose-intensified salvage radiation therapy is associated with low rates of acute grade 2 and $3 \mathrm{GU}$ and GI toxicity. ${ }^{24}$

Due to the biological characteristic of PC, an advantage in terms of benefits and toxicity (as well as in costs) can 
Table 3 Multivariate analysis (Cox model) for late toxicity

\begin{tabular}{llllll}
\hline Covariate & HR & SE & $\mathbf{z}$ & $\mathbf{p > z}$ & $\mathbf{9 5 \%}$ confidence interval \\
\hline RT+HT & 9.722 & 33.238 & 0.67 & 0.506 & $0.01 \mathrm{I}-7903.112$ \\
I+ Comorbidities & 0.036 & 0.065 & -1.85 & 0.064 & $0.001-1.206$ \\
Gleason score $>8$ & 0.032 & 0.048 & -2.26 & 0.024 & $0.001-0.636$ \\
I+ Positive lymph nodes & 5.565 & 19.662 & 0.49 & 0.627 & $0.005-5655.815$ \\
RT-salvage & 0.0157 & 0.058 & -1.13 & 0.260 & $0.00001-21.735$ \\
Pre-RT PSA & 0.138 & 0.218 & -1.25 & 0.210 & $0.006-3.048$ \\
Presurgery PSA & 1.135 & 0.095 & 1.51 & 0.131 & $0.962-1.340$ \\
Age & 1.169 & 0.099 & 1.83 & 0.067 & $0.988-1.382$ \\
\hline
\end{tabular}

Abbreviations: HR, hazard ratio; HT, hormonal therapy; PSA, prostate-specific antigen; RT, radiotherapy; SE, standard error of the mean.

A

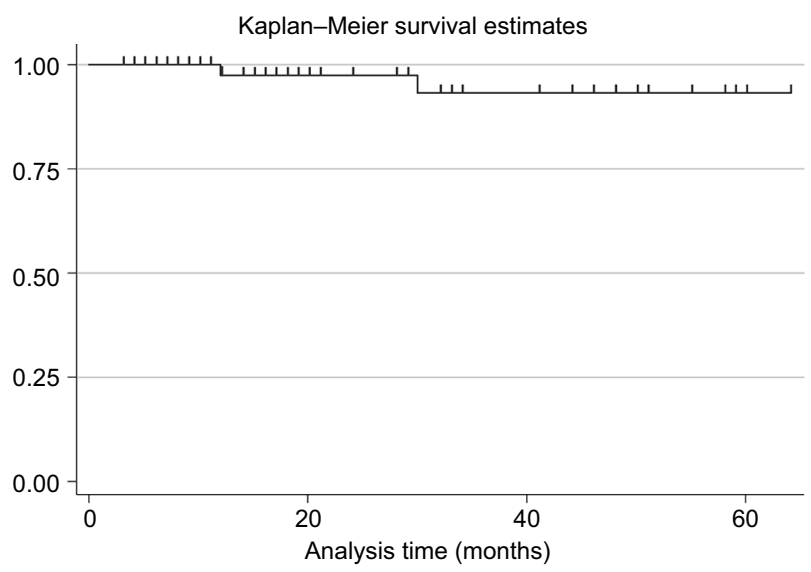

C

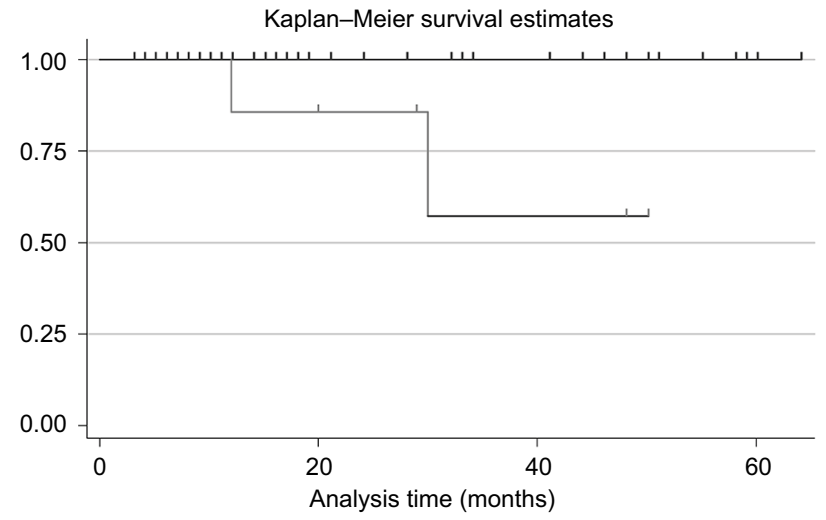

B

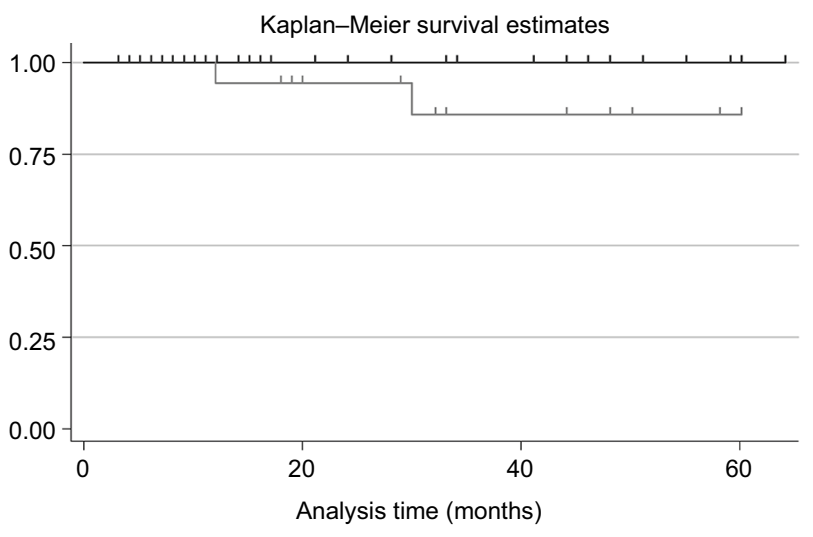

D

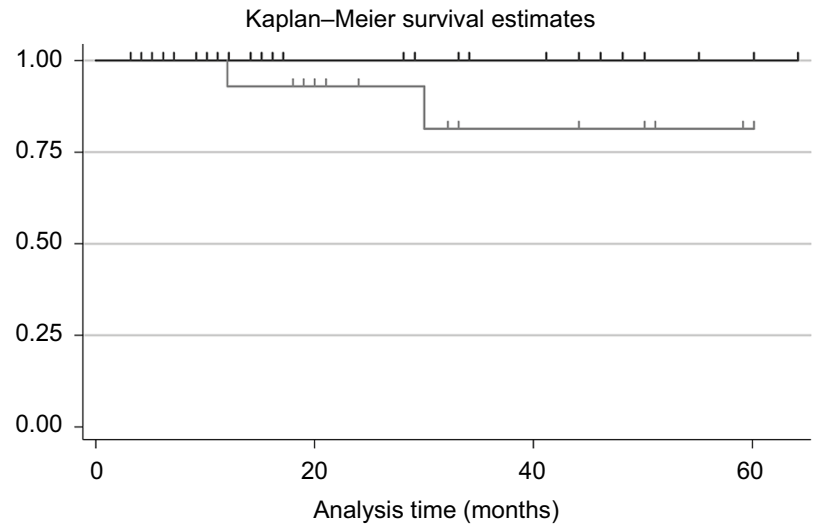

Figure 2 Kaplan-Meier survival estimates in the overall population (A), or stratified for: (B) treatment received (RT, black line vs RT+HT, gray line); (C) presence of at least one positive lymph node (0, black line vs I+, gray line); (D) PSA value ( $<10$, black line vs $\geq 10$, gray line).

Abbreviations: HT, hormonal therapy; PSA, prostate-specific antigen; RT, radiotherapy.

been obtained with hypofractionated RT. The available data on hypofractionated RT in adjuvant or salvage setting is mainly based on retrospective studies. ${ }^{8,25-28}$ In these studies, single doses from 2.4 to $3 \mathrm{~Gy}$, delivered with IMRT/ IGRT technique, were associated with low rates of grade 3 acute GI and GU toxicities. ${ }^{29}$ Alongi et al ${ }^{11}$ evaluated 172 patients undergoing whole-pelvis three-dimensional conformal radiotherapy (3D-CRT) versus IMRT and reported a lower risk of acute GI toxicity in the latter group. Similarly, another study ${ }^{30}$ showed a reduction in the risk of late grade 2 or higher GI toxicity in men receiving IMRT compared to those treated with 3D-CRT, even if IMRT was not associated 
with a reduction in risk of grade $\geq 2$ GU toxicity (5-yr IMRT, $16.8 \%$; 5-yr 3D-CRT, 15.8\%; $p=0.86)$, urinary incontinence (5-yr IMRT, 13.6\%; 5-yr 3D-CRT, 7.9\%; $p=0.25$ ), or grade 3 erectile dysfunction (5-yr IMRT, 26\%; 5-yr 3D-CRT, $30 \% ; p=0.82$ ). Cozzarini et $\mathrm{al}^{12}$ reported, after a median follow-up of 98 months, a 5-yr risk of late urinary toxicities of $6.9 \%$ and $18.1 \%$ in the conventionally fractionated and hypofractionated cohorts, respectively. In univariate analysis, the risk of late GU toxicity was predicted by dose per fraction, acute grade $\geq 2$ toxicity, and year of irradiation. On multivariate analyses, acute grade $\geq 2$ toxicity and dose per fraction independently predicted late adverse effects.

In the present analysis, although the follow-up is still short, the results of acute and late toxicity seem to confirm the feasibility of moderate hypofractionation when IMRT and IGRT are used.

There is also compelling evidence that adjuvant HT in combination with RT is indicated for patients with intermediate- or high-risk PC. The duration of HT depends on the risk category and can be short (4-6 months) for patients at intermediate risk and long ( 2 years and more) for patients with high risk. ${ }^{31-34}$

In this retrospective study, we also compared the effects of RT and RT+HT in patients undergoing RP in terms of OS and "toxicity-free" survival. The overall results are very positive with a high survival rate at 5 years $(>95 \%)$. Among the 64 patients studied, only two deaths occurred, both recorded in the RT+HT group. The high proportion of survival was associated with a low toxicity profile. Our study indicates the feasibility of hypofractionated RT due to its the low toxicity, which was only slightly increased when combined with HT.

The group of patients receiving the combination of RT and HT was the one with higher risk factors (a higher Gleason score, a higher level of presurgery PSA, and a presence of positive lymph nodes) compared to the group treated with RT only. The presence of high-risk factors justifies the addition of HT to RT and can explain the somehow increased risk of toxicity and slightly lower survival of this group relative to RT alone observed in the present study. It is to be pointed out that even in the presence of high negative prognostic factors, the OS of this group is still very good. The lack of a direct comparison in this study of a population with similar risk factors does not allow a proper comparison of the benefits of adding HT to RT. However, considering the high survival rate and the relatively low toxicity, it can be assumed that HT has an added value in terms of benefits for this category of patients.
Extrapolating some of the results of this study, it seems that for the acute toxicity, there is a trend of an increased risk of toxicity with age. This does not occur when late toxicity is considered. Since these results did not reach statistical significance, probably due to the small patient size, is difficult to speculate on their role, and so a more appropriate study on a larger population to specifically address these points is warranted.

\section{Conclusion}

In conclusion, we present evidence that in patients with PC undergoing RP, hypofractionated RT may contribute to achieve a high OS with an acceptable toxicity profile, even if a longer follow-up is needed to confirm these data. Combination of RT and HT is also well tolerated and efficacious.

\section{Disclosure}

The authors report no conflicts of interest in this work.

\section{References}

1. Global Burden of Disease Cancer Collaboration. Global, regional, and national cancer incidence, mortality, years of life lost, years lived with disability, and disability-adjusted life-years for 32 cancer groups, 1990 to 2015: a systematic analysis for the global burden of disease study. JAMA Oncol. 2016;3(4):524-548.

2. Bolla M, van Poppel H, Tombal B, et al. Postoperative radiotherapy after radical prostatectomy for high-risk prostate cancer: long-term results of a randomised controlled trial (EORTC trial 22911). Lancet. 2012;380(9858):2018-2027.

3. Thompson IM, Tangen CM, Paradelo J, et al. Adjuvant radiotherapy for pathological T3N0M0 prostate cancer significantly reduces risk of metastases and improves survival: long-term followup of a randomized clinical trial. J Urol. 2009;181(3):956-962.

4. Wiegel T, Bartkowiak D, Bottke D, et al. Adjuvant radiotherapy versus wait-and-see after radical prostatectomy: 10-year follow-up of the ARO 96-02/AUO AP 09/95 Trial. Eur Urol. 2014;66(2):243-250.

5. Brenner DJ. Hypofractionation for prostate cancer radiotherapy - what are the issues? Int J Radiat Oncol Biol Phys. 2003;57(4):912-914.

6. Cozzarini C, Fiorino C, Di Muzio N, et al. Hypofractionated adjuvant radiotherapy with helical Tomotherapy after radical prostatectomy: Planning data and toxicity results of a Phase I-II study. Radiother Oncol. 2008;88(1):26-33.

7. Fowler JF. The radiobiology of prostate cancer including new aspects of fractionated radiotherapy. Acta Oncol. 2005;44(3):265-276.

8. Katayama S, Striecker T, Kessel K, et al. Hypofractionated IMRT of the prostate bed after radical prostatectomy: acute toxicity in the PRIAMOS-1 trial. Int J Radiat Oncol Biol Phys. 2014;90(4):926-933.

9. Haustermans KMG, Hofland I, Van Poppel H, et al. Cell kinetic measurements in prostate cancer. Int J Radiat Oncol Biol Phys. 1997;37(5): $1067-1070$

10. Khoo VS, Pollack A, Cowen D, et al. Relationship of Ki-67 labeling index to DNA-ploidy, S-phase fraction, and outcome in prostate cancer treated with radiotherapy. Prostate. 1999;41(3):166-172.

11. Alongi F, Fiorino C, Cozzarini $\mathrm{C}$, et al. IMRT significantly reduces acute toxicity of whole-pelvis irradiation in patients treated with postoperative adjuvant or salvage radiotherapy after radical prostatectomy. Radiother Oncol. 2009;93(2):207-212. 
12. Cozzarini C, Fiorino C, Deantoni C, et al. Higher-than-expected severe (Grade 3-4) late urinary toxicity after postprostatectomy hypofractionated radiotherapy: a single-institution analysis of 1176 patients. Eur Urol. 2014;66(6):1024-1030.

13. Bria E, Cuppone F, Giannarelli D, et al. Does hormone treatment added to radiotherapy improve outcome in locally advanced prostate cancer? Cancer. 2009;115(15):3446-3456.

14. D'Amico AV, Moul J, Carroll PR, Sun L, Lubeck D, Chen MH. Cancerspecific mortality after surgery or radiation for patients with clinically localized prostate cancer managed during the prostate-specific antigen era. J Clin Oncol. 2003;21(11):2163-2172.

15. Sasse AD, Sasse E, Carvalho AM, Macedo LT. Androgenic suppression combined with radiotherapy for the treatment of prostate adenocarcinoma: a systematic review. BMC Cancer. 2012;12(1):54.

16. Donovan JL, Hamdy FC, Lane JA, et al. Patient-reported outcomes after monitoring, surgery, or radiotherapy for prostate cancer. $N \mathrm{Engl}$ J Med. 2016;375(15):1425-1437.

17. Roach M 3rd, Marquez C, Yuo HS, et al. Predicting the risk of lymph node involvement using the pre-treatment prostate specific antigen and gleason score in men with clinically localized prostate cancer. Int J Radiat Oncol Biol Phys. 1994;28(1):33-37.

18. Michalski JM, Lawton C, El Naqa I, et al. Development of RTOG consensus guidelines for the definition of the clinical target volume for postoperative conformal radiation therapy for prostate cancer. Int J Radiat Oncol Biol Phys. 2010;76(2):361-368.

19. Gladwish A, Loblaw A, Cheung P, et al. Accelerated hypofractioned postoperative radiotherapy for prostate cancer: a prospective phase I/ II study. Clin Oncol. 2015;27(3):145-152.

20. Tyldesley S, Peacock M, Morris JW, et al. The need for, and utilization of prostate-bed radiotherapy after radical prostatectomy for patients with prostate cancer in British Columbia. Can Urol Assoc J. 2012;6(2): 89-94.

21. Cozzarini C, Montorsi F, Fiorino C, et al. Need for high radiation dose (>or= $70 \mathrm{gy}$ ) in early postoperative irradiation after radical prostatectomy: a single-institution analysis of 334 high-risk, node-negative patients. Int J Radiat Oncol Biol Phys. 2009;75(4):966-974.

22. King CR. The timing of salvage radiotherapy after radical prostatectomy: a systematic review. Int J Radiat Oncol Biol Phys. 2012;84(1):104-111.

23. Ohri N, Dicker AP, Trabulsi EJ, Showalter TN. Can early implementation of salvage radiotherapy for prostate cancer improve the therapeutic ratio? A systematic review and regression meta-analysis with radiobiological modelling. Eur J Cancer. 2012;48(6):837-844.
24. Ghadjar P, Hayoz S, Bernhard Jr, et al. Acute toxicity and quality of life after dose-intensified salvage radiation therapy for biochemically recurrent prostate cancer after prostatectomy: first results of the randomized trial SAKK 09/10. J Clin Oncol. 2015;33(35):4158-4166.

25. Cozzarini C, Fiorino C, Da Pozzo LF, et al. Clinical factors predicting late severe urinary toxicity after postoperative radiotherapy for prostate carcinoma: a single-institute analysis of 742 patients. Int J Radiat Oncol Biol Phys. 2012;82(1):191-199.

26. Fersino S, Tebano U, Mazzola R, et al. Moderate hypofractionated postprostatectomy volumetric modulated arc therapy with daily image guidance (VMAT-IGRT): a mono-institutional report on feasibility and acute toxicity. Clin Genitourin Cancer. 2017;15(4):e667-e673.

27. Kruser TJ, Jarrard DF, Graf AK, et al. Early hypofractionated salvage radiotherapy for post-prostatectomy biochemical recurrence. Cancer. 2011;117(12):2629-2636.

28. Massaccesi M, Cilla S, Deodato F, et al. Hypofractionated intensitymodulated radiotherapy with simultaneous integrated boost after radical prostatectomy: preliminary results of a phase II trial. Anticancer Res. 2013;33(6):2785-2789.

29. Azelie C, Gauthier ML, Mirjolet CL, et al. Exclusive image guided IMRT vs. radical prostatectomy followed by postoperative IMRT for localized prostate cancer: a matched-pair analysis based on risk-groups. Radiat Oncol. 2012;7(1):158.

30. Goenka A, Magsanoc JM, Pei X, et al. Improved toxicity profile following high-dose postprostatectomy salvage radiation therapy with intensity-modulated radiation therapy. Eur Urol. 2011;60(6):1142-1148.

31. Bolla M, Van Tienhoven G, Warde P, et al. External irradiation with or without long-term androgen suppression for prostate cancer with high metastatic risk: 10-year results of an EORTC randomised study. Lancet Oncol. 2010;11(11):1066-1073.

32. Horwitz EM, Bae K, Hanks GE, et al. Ten-year follow-up of radiation therapy oncology group protocol 92-02: a phase III trial of the duration of elective androgen deprivation in locally advanced prostate cancer. J Clin Oncol. 2008;26(15):2497-2504.

33. Pisansky TM, Hunt D, Gomella LG, et al. Duration of androgen suppression before radiotherapy for localized prostate cancer: radiation therapy oncology group randomized clinical trial 9910. J Clin Oncol. 2015;33(4):332-339.

34. Zilli T, Dal Pra A, Kountouri M, Miralbell R. Prognostic value of biochemical response to neoadjuvant androgen deprivation before external beam radiotherapy for prostate cancer: a systematic review of the literature. Cancer Treat Rev. 2016;46:35-41.
Cancer Management and Research

\section{Publish your work in this journal}

Cancer Management and Research is an international, peer-reviewed open access journal focusing on cancer research and the optimal use of preventative and integrated treatment interventions to achieve improved outcomes, enhanced survival and quality of life for the cancer patient. The manuscript management system is completely online and includes

\section{Dovepress}

a very quick and fair peer-review system, which is all easy to use. Visit http://www.dovepress.com/testimonials.php to read real quotes from published authors. 